\title{
MobileWars: A Mobile GPGPU Game
}

\author{
Mark Joselli ${ }^{1}$, Jose Ricardo Silva Jr. ${ }^{2}$, Esteban Clua, and Eduardo Soluri ${ }^{3}$ \\ 1 PUC-PR \\ mejoselli@gmail.com \\ 2 MediaLab - UFF \\ \{jricardo, esteban\}@ic.uff.br \\ 3 Nullpointer Tecnologia \\ esoluri@nullpointer.com.br \\ http://www.nullpointer.com.br
}

\begin{abstract}
Nowadays mobile phones, especially smartphones, are equipped with advanced computing capabilities. Most of these devices have multicore processors such as dual-core CPUs and many-core GPUs. These processors are designed for both low power consumption and high performance computation. Moreover, most devices still lack libraries for generic multicore computing usage, such as CUDA or OpenCL. However, computing certain kind of tasks in these mobile GPUs, and other available multicores processors, may be faster and much more efficient than their single threaded CPU counterparts. This advantage can be used in game development to optimize some aspects of a game loop and also include new features. This work presents an architecture designed to process most of the game loop inside a mobile GPU using the Android Renderscript API. As an illustrated test case for the proposed architecture, this work presents a game prototype called "MobileWars", completely developed using the proposed architecture.
\end{abstract}

Keywords: Mobile, Mobile Multicore Computing, Game Architecture, GPGPU, Game Physics, Game AI, RenderScript API, Android.

\section{Introduction}

Digital games are defined as real-time multimedia applications that have a time constraints to run their tasks [1]. If the game is not able to execute its processing under some time threshold, it will fail [2, as its immersion will be lost. Mobile games are also real-time multimedia application that run on mobile phones, having the same time constraints. However, many others characteristics are different [3, when compared to PC or console games: hardware (processing power and screen size); user input, (buttons, voice, touch screen and accelerometers); and a big diversity of operating systems, such as Android, iOS, Symbian and Windows Mobile 4.

The market of mobile devices is growing [5]. Devices powered with Android have $60 \%$ of the sale market share in the first quarter of this year in the USA, according to [6]. Also the usage of the internet on such devices are gaining 
importance, since its has been doubling every year 7]. These are important motivations for game developers and designer to create blockbusters and high end mobile games.

Google introduced in the Honeycomb version of Android the Renderscript API (application programming interface) [8]. Renderscript is an API for achieving better performance on Android phones and tablets. Using this API, developers can use the same code to process on different hardware architectures such as different CPUs (Central Processing Unity), ARM (Advanced RISC Machine) v5, ARM v7, and X86, GPUs (Graphic Processing Unit) and DSPs (Digital Signal Processors). The API decides which processor will run the code on the device at runtime, choosing the best processor for the available code. This work presents a novel game architecture suitable for this new architecture, which extends previous work that were applied in a desktop hardware [2. As far as the authors knows, this is the first mobile game that uses this kind of architecture.

Summarizing, this work has following contributions: Modeling of a game architecture for mobile device; Adaptation of the architecture and data structures for Renderscript API; and Implementation of a state machine on the mobile GPU.

The paper is organized as follows: Section 2 presents related works on mobile multicore processing and the Renderscript API. Section 3 presents the related work on GPGPU on mobile devices while Section 4 the game design of MobileWars is presented. Section 5 presents the game architecture used in this work and Section 6 presents the results. Finally Section 7 presents the conclusion of this paper.

\section{Mobile Multicore Processing}

Multicore architecture are, nowadays, found in home PCs and mobile phone, available as multicore CPUs and GPUs. GPUs are powerful processors originally dedicated to graphics computation 9]. GPUs for PCs are composed by hundreds of parallel processors, achieving much better performance then modern CPUs for several specific applications scenarios. The GPU can be used on the PC as a generic processor to process data and deal with computationally intensive tasks, through development of elaborate architectures such as CUDA (Compute Unified Device Architecture ) and OpenCL.

On mobile devices, the GPU is much less capable and powerful [10, and is typically integrated into the mobile processor system-on-a-chip (SoC), which also consists of one or several CPUs, DSP (digital system processor), and other available mobile-specific accelerators. This embedded GPU does not have a dedicated memory, having to share the system bus with others processors for accessing the memory. Consequently the memory bandwidth is also much lower when compared to desktops GPUs [11. Currently, mobile GPUs emphasis more on lower power consumption [12] than performance. Some of these currently available GPUs devices are the Qualcomm's Adreno 200, the TI's PowerVR SGX 530/535 and the nVidia Tegra3. 
Normally, most works that uses mobile for parallel processing deals with the use of GPU for generic processing with the OpenGL ES programable shaders, the vertex and fragment shader, as the programming interface [13]. The disadvantage found in some approaches is the traditional shader languages limitations (such as scatter memory operations, i.e. out-of-order indexed write array operations), and features that are not even implemented on those languages (such as integer data operands like bit-wise logical operations AND, OR, XOR, NOT and bitshifts). Some of these disadvantages are also presented in the Rendersript API, as the limitation of scatter memory operations.

\subsection{The Renderscript API}

Renderscript is a new software development kit and API for Android firstly introduced by Google in the Honeycomb version of Android. Renderscript is an API for high-performance graphics processing on Android phones and tablets. It is used for fast 3D rendering and computing processing, having similar paradigm as GPU computing libraries and frameworks [14. The main goal of Renderscript API is to bring a lower level, higher performance API to Android developers, in order to achieve better performance on visual animations and simulations [15].

Renderscript code is compiled at runtime on the device, avoiding the necessity of application recompilation for different processor types. This fact makes its usage more easily for the developer. Its language is an extension of the C99 language that is translated to an intermediate bytecode at compile time and then to machine code at runtime. The API scales the generated code to the amount of processing cores available on the device, being it CPUs, GPUs or DSPs. The decision of choosing which processor is going to run the code is made on the device at runtime, being completely transparent for the developer. Normally simple scripts will be able to run on the available GPUs, while more complex scripts will run on the CPU. The CPU is also a fallback, running the code in case there is no available suitable device.

All the tasks implemented in Renderscript are automatically portable for parallel processing on the available processors of the device, such as the CPU, GPU and even DSP. Renderscript is specially useful for apps that do image processing, mathematical modeling, or any other operation that requires lots of mathematical computation, similar to GPU computing paradigm. The main use of Renderscript is to gain performance in critical code where the traditional Android framework and OpenGL ES APIs are not fast enough.

\section{Related Work}

There are few works on literature about approaches that deals with the use of the mobile on multicore processors, especially dealing with games. Most of the work deals with image processing, using the GPU for generic processing. In [16] some image processing algorithms are designed and implemented on handheld device using OpenGL ES 2.0. In [17] a mobile-GPU implementation of Local 
Binary Pattern feature extraction is presented, showing a better performance and power consumption when used the CPU together with the GPU.

Also using OpenGL 2.0 for image processing [11] shows a face recognition algorithm, with a $4.25 \mathrm{x}$ speedup and a $3.88 \mathrm{x}$ reduction on the total energy consumption. 18 presents an implementation of GPU-based window system on top of EGL and OpenVG. Also the openCV [19] is a library for computer vision, which includes some new and experimental features for the mobile devices. These works are particularly important since they show that the use of the mobile GPU is faster and have low power consumption.

Nah et al. 20] shows OpenGL ES-based CPU-GPU hybrid ray tracer for mobile devices, using Kd-trees. In [21, a system for building document mosaic images from selected video frames on mobile phones using the GPU for accelerating its processing is presented. Also a image deformation implementation with a misc of ARM-Linux and OpenGL ES for mobile device is presented in [22].

There are also some works 23242425 26] that uses cloud-computing for distribution of the processing over the cloud for mobile in real time simulation and games. These approaches tend to rely on the network for these distribution, which can be very unreliable and slow using the mobile phones carrier.

Unfortunately, there is nothing in literature that deals with game tasks processed on the mobile GPGPU such as proposed in this work. The only similar work is the previous one [27, where a flocking boids is implemented in the mobile GPU using the Renderscript. This work also takes some concepts from [2], where a PC game were fully implemented in the GPU using CUDA.

\section{Design of the Game}

The MobileWars game prototype is a massive 2D prototype shooter with a topdown perspective. The game is similar to games like Geometric Wars and E4. The main enhancements of MobileWars is that it uses the mobile GPU to process its calculations, allowing to process and render thousands of enemies, while similar games only process hundreds.

The game play is very simple: the player plays as a mobile phone (which is called "mShip") inside the "computer universe", where the main task is to process (by shooting them) polygons, shaders and data (the enemies) from a game. Every time the "mShip" make physical contact with a enemy it looses time and in consequence it looses FPS. The objective is to process the maximum number of data in the smaller amount of time, at the same time keeping the game interactive with a minimum 12 frames per second. The MobileWars uses the multi-touch screen as the input device of the game, controlling the movement of the "mShip", and the direction of the shots.

\section{Proposed Architecture}

Computer games are multimedia applications that employ knowledge of many different subjects, such as Computer Graphics, Artificial Intelligence, Physics, 
Network and others 28. Additionally, computer games are also interactive applications that exhibit three general classes of tasks: data acquisition, data processing, and data presentation. Data acquisition in games is related to gathering data from input devices like touch, mice and accelerometers. Data processing consist on applying game rules in order to responding user commands, simulating Physics and Artificial Intelligence behaviors. Data presentation is relate to provides feedback to the player about the current game state, usually through image, audio, and force feedback. In the proposed architecture, all game logic is processed in GPU, i.e., all the data processing tasks. The CPU is only used for tasks that cannot be archived in a parallel manner, such as data acquisition.

In order to fully work on the mobile device, the simulation architecture is divided into four different ambients: the Android framework, where the application is created together with the renderscript context. Also, this ambient is responsible for gathering inputs and to sends them to computing renderscript; the computing renderscript is where the variables for the simulation are created, the call for the renderscript computing engine are made and the maintenance of the NGrid, which is a data structure for neighborhood gathering, which will be explained in the next subsection. The renderscript computing engine process the behavior of the scene, distributing its process among the available processors. OpenGL is responsible to render all the objects, applying shaders and visual effects on them. This architecture is illustrated in Figure 1.

The execution of the MobileWars work as follows. First the Activity create all the context in order to use the RenderScript API. Then the compute Renderscript initialize all the necessary variables and creates the player and enemies. After this initialization, the game loop starts by gathering the user input throughout the Activity and sending it to the Compute Renderscript for further processing. The Compute Renderscript make the calls to the Renderscript Compute Engine for the processing of the game physics and game AI in all entities of the game. The Compute Renderscript does a process based on the rules of the NGrid data structure. All these updates are stored on a VBO (Vertex Buffer Object) and sent to the shaders for its rendering. The GPU also share variables with CPU in order to tell whether it should terminate the application or play sounds effects.

Renderscript programs are divided in threads. In order to process the main game logic, which needs to be executed sequentially, the proposed architecture have a special thread which is responsible for it. This thread is also responsible to treat the "mShip" data and inputs. In this process, the following tasks are included: update the position of the "mShip" accordingly to the input; creation of shots, which are made by other threads; computing the scores; checks the game over; and check for the creation of new enemies. The others threads are responsible for updating the enemies (as a physisical-behavior entity), the shots (as a physical entity) and some particles animations, like explosions (as a behavior entity). The positions and type are put in a VBO and sent to a vertex shader in order to render the individuals without using the Activity. 


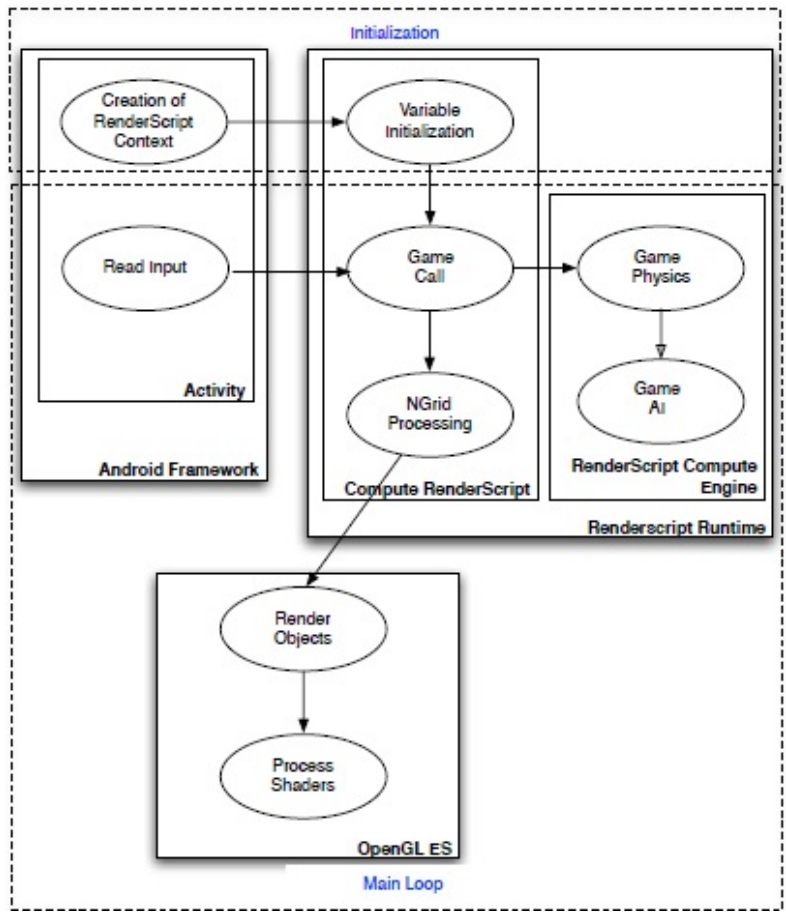

Fig. 1. Architecture Overview

Since all the entities are being updated and executed using the GPU, which has some limitation. It is important to have a data structure that is efficient and capable of adapting in such hardware. In order to fulfill this need this work uses the NGrid, which is presented in the next subsection.

\subsection{The NGrid}

In order to process the physics and AI, some sort of neighborhood gathering is needed. Most of the games tries to avoid the high complexity of proximity queries by applying some kind of spatial subdivision to the environment and classifying entities among the cells based on their position. To accelerate data fetching, the entities list must be sorted in such a way that all entities on the same cells are grouped together. This approach helps lowering the number of proximity queries but is very sensible to the maximum number of entities that can fit in a single cell. The proposed architecture implements a novel simulation data structure that maintains entities into another kind of proximity based structure, which is called NGrid. In this data structure, each cell now fits only one entity and does not directly represent a discrete spatial subdivision. The NGrid is an approximate representation of the system of neighborhoods in the environment that maps the $\mathrm{N}$-dimensional environment to a discrete map (lattice) with $\mathrm{N}$ 


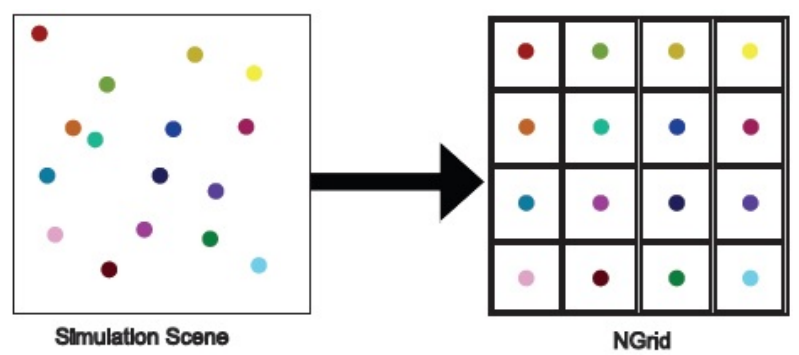

Fig. 2. Construction of the NGrid in a top-down camera

dimensions. This way, entities that are close in a neighborhood sense appear close to each other in the map. Thus can be also seen as a multi-dimensional compression of the environment that still keeps the original position information of all entities. Figure 2 shows the contraction of the NGrid.

In order to keep entities that are close in a neighborhood sense to appear close to each other in the NGrid, a sorting mechanism is used. Since the entities moves at each frame, the neighborhood grid becomes misaligned. In order to maintain the neighborhood grid in such a way that neighbors in geometric space are stored in cells close to each other, the sorting needs to be done at every step. For this work a odd-even sort data structure is used. More on this data structure can be seen on 2930.

\subsection{Physics Processing}

The Physics processing is responsible for the update of physical behavior, i.e, how the entities process and resolve all collisions and forces responses. The Physics of this architecture is based on the one found for particle systems 31 and a hybrid physics engine [28].

Collision detection is a complex operation. For $n$ entities in a system, there must be a collision check between the $O\left(n^{2}\right)$ pairs of entities. Normally, to reduce this computation cost, this process is performed in two steps: first, the broad phase, and second, the narrow phase. In the broad phase, the collision library detects which bodies have a chance of collision among themselves, where the NGrid is being used. In the narrow phase, a more refined algorithm to do the collisions tests are performed between the pairs of bodies that had passed in the broad phase.

The Physics step is responsible for making the broad phase of the collision detection; it also executes the narrow phase of the collision detection, i.e, apply the collision in each body; forwarding the simulation step for each body by computing the new position and velocities according to the their forces and the time step by integrating the equations of motion. 


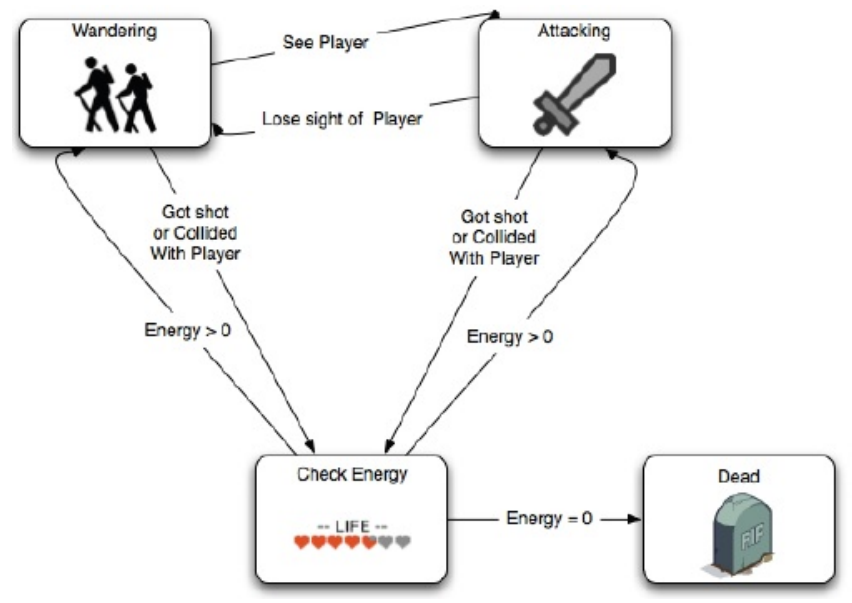

Fig. 3. A Example of State Machine

\subsection{Game AI and Login}

Game AI is used to produce the illusion of intelligence in the behavior of nonplayer characters (NPC) which is applied for enemies, in most games. There are a collection of techniques used to implement the game AI such as finite state machines, fuzzy logic, neural networks, and many others. This work uses finite state machine (FSM), which are powerful tools used in many computer game implementations [32, like the NPC behavior, the characters animation states and the game menu states. A finite state machine is a model of behavior composed of states, transitions between those states, and the actions.

The behaviors are affected by the size of vision (which uses the NGrid), velocity and energy, being variables available for each type of enemy. With the modification of these values, this work implements different types of enemies, all based on state machines, as detailed in the following subsections. The following behaviors are proof of concepts, which could be changed or customized in order to the specific game project.

Kamikaze Behavior. The kamikaze approach is a behavior that simulates suicidal attacks. It wanders until the "mShip" is saw, which attacking it by throwing itself against it. This approach is well suited for a parallel architectures, since few information about the scene is necessary. It uses a state machine that has only four state, wandering, attacking, checking energy and dead. This state machine can be seen can be seen on Figure 3 .

Group Behavior. The group behavior is a behavior that make groups, avoid bullets and attacks. The individual wanders try to find similar individuals, i.e, individuals of the same type, and the "mShip". If it sees a similar individual, it 
goes close to it and make a group. And if it can see the player, it attacks the player by throwing itself against it. If the individual sees a bullet coming in its direction it tries to avoid. It has a state machine that has six state, wandering, grouping, attacking, checking energy, avoiding bullets and dead.

Tricky Behavior. The tricky behavior is the most complex one found in the game. This behavior, besides trying to group similar individuals, it also is the only that recover energy. At first, the enemy wanders trying to find the "mShip" or similar individuals. If it sees a similar individual, it goes close to it and make a group. In case it sees the player, it throws itself against it. Additionally, if the individual sees a bullet coming in its direction, it tries to avoid it. Finally, in case of low energy, it tries to scape to recover the lost energy. Their state machine has seven states: wandering, grouping, attacking, avoiding bullets, checking energy, escaping, and dead.

\section{Results}

For the tests, we have used an Asus Tranformers TF101, which is a 10.1 inches tablet with Android 4.1.1 as operating system. Also, it has a Nvidia Tegra 2 T20 chipset with a Dual-core $1 \mathrm{GHz}$ Cortex-A9 CPU and a ULP (ultra-low power) GeForce GPU, with 8 cores and 1GB of RAM memory. Simulations tests with different configurations were performed. The rendering is done in screen space, through applying a bilateral filter in sphere's normal. To assure that results are consistent, each test was repeated 10 times and the standard deviation of the average times was confirmed to be within $5 \%$.

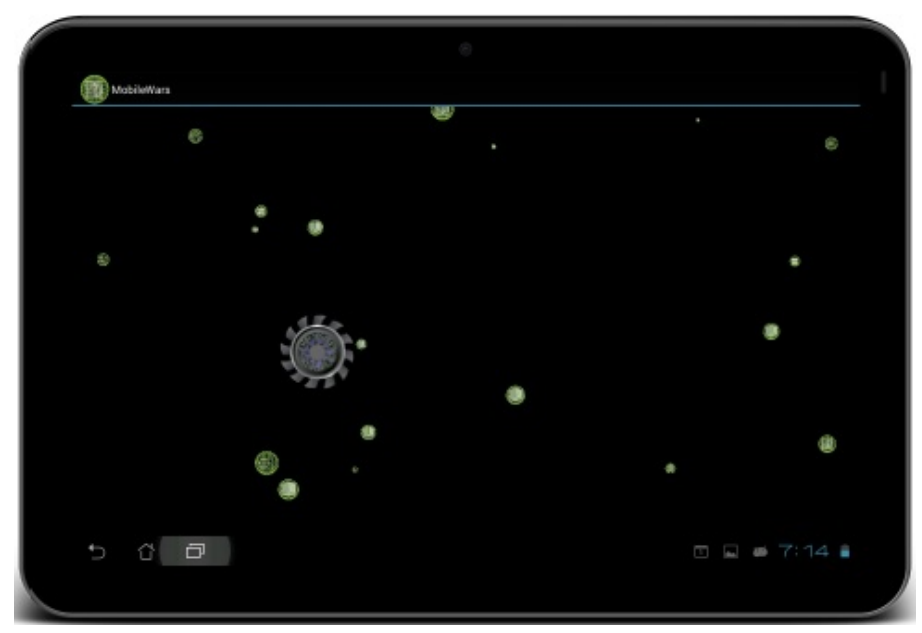

Fig. 4. A screenshot of the game in execution 
The number of enemies determines the performance of the game. This work has decided to have a maximum bound of 8192 enemies. A screenshot of the game can be seen in Figure 4.

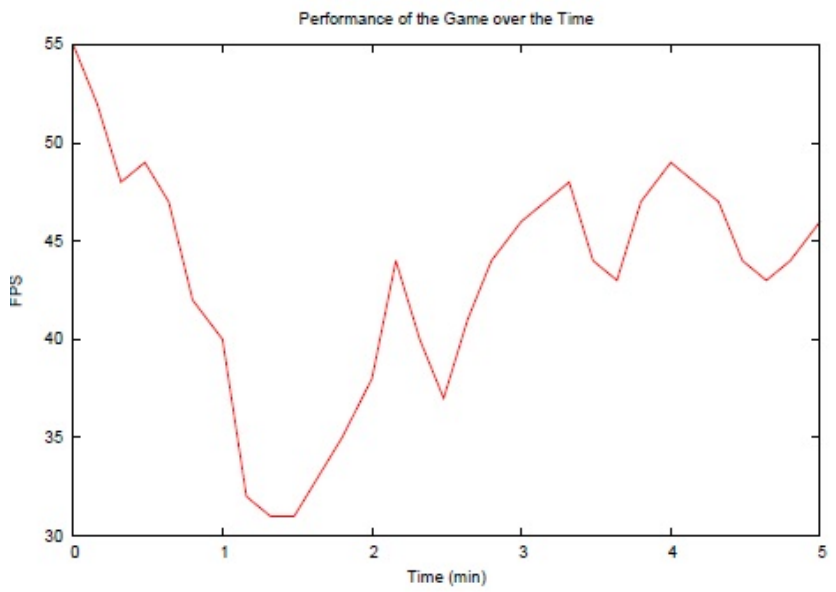

Fig. 5. Measured performance of the game

Similar to others game of the same type, the game has waves of enemies, where a huge amount of enemies appear in a short period of time. Figure 5 show a graph presenting the performance, in FPS, of the game in 5 minutes execution, where a huge wave of $8 \mathrm{k}$ enemies appeared during the first minute of the test.

From Figure 5, it can be seen that the game FPS varies from 50 to 30 FPS during the game. The achieved performance is considered optimal in a game 33. The proposed game prototype has been tested without the architecture in two ambients: using the Android framework and the native code. The game FPS varies from 17 to 5 FPS (the presented architecture has at least $3 \mathrm{x}$ speedup) with the android framework. Using the native code, the game FPS varies from 13 to 27 FPS (the presented architecture has a minimum of $2 \mathrm{x}$ speedup).

\section{Conclusions}

The mobile phones have evolved to high end devices with GPUs that can be used to process different tasks of the game loops. The proposed architecture presents a game that uses this kind of devices for processing the whole game loop. The presented results can make a new trend on game development, as more processing can be done in the same period of time. Using the presented architecture, a game could achieve a minimum $2 \mathrm{x}$ speedup against native code, and a 3x speedup against the traditional Android framework. As future work, we are focusing on creating a more generic framework in order to be used in others game types. 


\section{References}

1. Joselli, M., Zamith, M., Clua, E., Leal-Toledo, R., Montenegro, A., Valente, L., Feijo, B., Pagliosa, P.: An architeture with automatic load balancing for real-time simulation and visualization systems. JCIS - Journal of Computational Interdisciplinary Sciences, 207-224 (2010)

2. Joselli, M., Clua, E.: Gpuwars: Design and implementation of a gpgpu game. In: Brazilian Symposium on Games and Digital Entertainment, pp. 132-140 (2009)

3. Joselli, M., Silva Jr., J.R., Zamith, M., Soluri, E., Mendonca, E., Pelegrino, M., Clua, E.W.G.: An architecture for game interaction using mobile. In: 2012 IEEE International Games Innovation Conference (IGIC), pp. 73-77 (August 2012)

4. Joselli, M., Clua, E.: Grmobile: A framework for touch and accelerometer gesture recognition for mobile games. In: Proceedings of the 2009 VIII Brazilian Symposium on Games and Digital Entertainment, SBGAMES 2009, pp. 141-150. IEEE Computer Society, Washington, DC (2009)

5. Koivisto, E.M.I.: Mobile games 2010. In: CyberGames 2006: Proceedings of the 2006 International Conference on Game Research and Development, pp. 1-2. Murdoch University, Australia (2006)

6. CNET: Android reclaims 61 percent of all u.s. smartphone sales (2012), http://tinyurl.com/cgsszfc

7. GlobalStats: Mobile internet usage is doubling year on year (2012), http://gs.statcounter.com/press/ mobile-internet-usage-is-doubling-year-on-year

8. Android, G.: Android renderscript (2012), http://developer.android.com/guide/topics/renderscript/index.html

9. Joselli, M., Silva Jr., J.R., Zamith, M., Soluri, E., Mendonca, E., Pelegrino, M., Clua, E.W.G.: Techniques for designing gpgpu games. In: 2012 IEEE International Games Innovation Conference (IGIC), pp. 78-82 (August 2012)

10. Akenine-Moller, T., Strom, J.: Graphics processing units for handhelds. Proceedings of the IEEE 96, 779-789 (2008)

11. Cheng, K.T., Wang, Y.C.: Using mobile gpu for general-purpose computing a case study of face recognition on smartphones. In: 2011 International Symposium on VLSI Design, Automation and Test (VLSI-DAT), pp. 1-4 (2011)

12. Therdsteerasukdi, K., Byun, G., Cong, J., Chang, M.F., Reinman, G.: Utilizing rf-i and intelligent scheduling for better throughput/watt in a mobile gpu memory system. ACM Trans. Archit. Code Optim. 8, 51:1-51:19 (2012)

13. Kim, T.-Y., Kim, J.-H., Hur, H.: A unified shader based on the openGL ES 2.0 for 3D mobile game development. In: Hui, K.-c., Pan, Z., Chung, R.C.-k., Wang, C.C.L., Jin, X., Göbel, S., Li, E.C.-L. (eds.) EDUTAINMENT 2007. LNCS, vol. 4469, pp. 898-903. Springer, Heidelberg (2007)

14. Huang, Y., Chapman, P., Evans, D.: Privacy-preserving applications on smartphones. In: Proceedings of the 6th USENIX Conference on Hot Topics in Security, HotSec 2011, p. 4. USENIX Association, Berkeley (2011)

15. Guihot, H.: Pro Android Apps Performance Optimization. Apress (2012)

16. Singhal, N., Yoo, J.W., Choi, H.Y., Park, I.K.: Design and optimization of image processing algorithms on mobile gpu. SIGGRAPH Posters, 21 (2011)

17. Lopez, M., Nykänen, H., Hannuksela, J., Silven, O., Vehviläinen, M.: Accelerating image recognition on mobile devices using gpgpu. In: Proceedings of SPIE, vol. 7872 , p. $78720 \mathrm{R}(2011)$ 
18. Jeong, D., Kamalneet, Kim, N., Lim, S.: Gpu-based x server on top of egl and openvg. In: International Conference on Computers in Education, pp. 1-2 (2009)

19. Pulli, K., Baksheev, A., Kornyakov, K., Eruhimov, V.: Real-time computer vision with opencv. Commun. ACM 55, 61-69 (2012)

20. Nah, J.-H., Kang, Y.-S., Lee, K.-J., Lee, S.-J., Han, T.-D., Yang, S.-B.: Mobirt: an implementation of opengl es-based cpu-gpu hybrid ray tracer for mobile devices. In: ACM SIGGRAPH ASIA 2010 Sketches, SA 2010, pp. 50:1-50:2. ACM, New York (2010)

21. López, M.B., Hannuksela, J., Silvén, O., Vehvilainen, M.: Graphics hardware accelerated panorama builder for mobile phones. In: Proc. SPIE Multimedia on Mobile Devices, vol. 7256 (2009) ISBN 9780819475060

22. Hu, X., Xia, Z., Yuan, Z.: Study on image deformation simulation based on arm linux and opengles. In: Proceedings of the 2011 International Conference on Intelligence Science and Information Engineering, ISIE 2011, pp. 303-306. IEEE Computer Society, Washington, DC (2011)

23. Barboza, D.C., Junior, H.L., Clua, E.W.G., Rebello, V.E.: A simple architecture for digital games on demand using low performance resources under a cloud computing paradigm. In: Brazilian Symposium on Games and Digital Entertainment, pp. 33-39 (2010)

24. Joselli, M., Zamith, M., Clua, E.W.G., Montenegro, A., Leal-Toledo, R.C.P., Valente, L., Feijó, B.: An architecture with automatic load balancing and distribution for digital games. In: 2010 Brazilian Symposium on Games and Digital Entertainment (SBGAMES), pp. 59-70. IEEE (2010)

25. Zamith, M., Valente, L., Joselli, M., Clua, E., Toledo, R., Montenegro, A., Feij, B.: Digital games based on cloud computing. In: SBGames 2011 - X Simpsio Brasileiro de Jogos Para Computador e Entretenimento Digital (2011)

26. OnLive (2012), http://www.onlive.com/

27. Joselli, M., Soluri, E., Passos, E., Junior, J.S., Zamith, M., Clua, E.: A flocking boids simulation and optimization structure for mobile multicore architectures. In: SBGames 2012 - Trilha de Computao, Braslia (2012)

28. Joselli, M., Clua, E., Montenegro, A., Conci, A., Pagliosa, P.: A new physics engine with automatic process distribution between cpu-gpu. In: Sandbox 2008: Proceedings of the 2008 ACM SIGGRAPH Symposium on Video Games, pp. 149-156 (2008)

29. Joselli, M., Passos, E.B., Zamith, M., Clua, E., Montenegro, A., Feijo, B.: A neighborhood grid data structure for massive $3 \mathrm{~d}$ crowd simulation on gpu. In: Brazilian Symposium on Games and Digital Entertainment, pp. 121-131 (2009)

30. Passos, E.B., Joselli, M., Zamith, M., Clua, E.W.G., Montenegro, A., Conci, A., Feijo, B.: A bidimensional data structure and spatial optimization for supermassive crowd simulation on gpu. Comput. Entertain. 7, 60:1-60:15 (2010)

31. Joselli, M., Zamith, M.: A novel data structure for particle system simulation based on gpu with the use of neighborhood grids. In: Proceedings of the GPU Forum 2012 (CSBC), SBC (2012)

32. Li, F., Woodham, R.J.: Video analysis of hockey play in selected game situations. Image Vision Comput. 27, 45-58 (2009)

33. Joselli, M., Zamith, M., Valente, L., Clua, E.W.G., Montenegro, A., Conci, A., P., F.P.: An adaptative game loop architecture with automatic distribution of tasks between cpu and gpu. In: Proceedings of the VII Brazilian Symposium on Computer Games and Digital Entertainment, pp. 115-120 (2008) 